\title{
Liquid-liquid extraction and ultrafiltration based sample preparation technique for Q-TOF LC/MS analysis of non- polar metabolites in human plasma samples
}

\author{
Engin KOÇAK 1,a $\mathbb{D}$, Ozan KAPLAN 1,a (D), Mustafa ÇELEBİER $1 *$ (D) \\ 1 Department of Analytical Chemistry, Faculty of Pharmacy, Hacettepe University, Ankara, Turkey. \\ * Corresponding Author. E-mail: celebier@hacettepe.edu.tr (M.Ç.), Tel. +90-0312-305 1499. \\ a These authors equally contributed to the presented work.
}

Received: 15 March 2019 / Revised: 09 August 2019 / Accepted: 10 August 2019

\begin{abstract}
Metabolomics is one of the main areas to understand cellular process at molecular level by analyzing metabolites. In recent years, metabolomics has been emerged as key tool to understand molecular basis of disease, find diagnostic and prognostic biomarkers, and develop new treatment opportunities. One of the most important challenge for metabolomics analysis is sample complexity due to wide concentration dynamics. In untargeted metabolomics studies for human plasma samples, generally there is no pre-fractioning method for metabolites prior to LC/MS analysis. This situation causes problem for analysis of some specific metabolites belonging to different pathways in such a complex system. In this study, it was offered liquid-liquid extraction and ultrafiltration-based sample preparation technique for fractioning of non-polar metabolites. Human plasma metabolites were extracted with a well-known cosolvent system (methanol/water/chloroform) and separated according to their polarity in aqueous and organic phases. Ultrafiltration process was performed for both phases to clean-up the sample. Solvents were evaporated and the remaining parts were dissolved in an organic solvent, acetonitrile, to obtain the non-polar metabolite mixtures. Samples were analyzed using Q-TOF LC/MS system for metabolite profiling. Metabolites were separated in a C18 (Agilent Zorbax C18 $1.8 \mu \mathrm{M}, 50 \times 2.1 \mathrm{~mm}$ ) column at $0.200 \mathrm{ml} \mathrm{min}^{-1}$ flow rate. Water and acetonitrile mixture including $0.1 \%$ formic acid was used as the mobile phase in a $25 \mathrm{~min}$ gradient elution system. In the aqueous phase, 249 peaks were found and 60 peaks were obtained in the organic phase. 14 peaks were common in both phases. Results showed that co- solvent system could be used for pre-fractioning of metabolites and reduce complexity of human plasma for metabolite profiling.
\end{abstract}

KEYWORDS: Metabolomics; human plasma; XCMS; liquid-liquid extraction; ultrafiltration.

\section{INTRODUCTION}

Metabolomics is the vast scale investigation of little particles, generally known as metabolites, inside cells, bio fluids, tissues or life forms. These little particles and their connections inside an organic framework are known as the metabolome. Similarly, as genomics is the investigation of DNA and hereditary data inside a cell, and transcriptomics is the investigation of RNA and contrasts in mRNA articulation; metabolomics is the investigation of substrates and results of digestion, which are impacted by both hereditary and ecological components. Metabolomics is an intense methodology since metabolites and their fixations, dissimilar to other "omics" measures, straightforwardly mirror the basic biochemical movement and condition of cells/tissues. Accordingly, metabolomics best speaks to the molecular phenotype [1].

The metabolomics studies can be arranged into two classes, which are targeted studies and untargeted studies known as metabolite profiling. Untargeted studies are for absolute determination of already focused metabolites while metabolite profiling is to compare relative amount of the metabolites changed according to environmental effects like age, diet and diseases [2]. For targeted studies, the analytical procedure should have high accuracy, precision and selectivity to determine the focused metabolites in the samples. The results are obtained by using the standards of the metabolites. Data mining procedure is to compare the absolute amount of some metabolites known as biomarkers or the metabolites in a pathway related with the subject of investigation. Thus, the analytical results clearly show the differences within each group. However, fit-forreason logical strategies are produced to get information on relative comparison of wide range of metabolites

How to cite this article: Koçak E, Kaplan O, Çelebier M. Liquid-liquid extraction and ultrafiltration based sample preparation technique for Q-TOF LC/MS analysis of non-polar metabolites in human plasma samples. J Res Pharm. 2020; 24(1): 38-43. 
for untargeted analyses. Data acquisition for untargeted metabolomics is to handle with metabolites having no specific knowledge of them whether they will be detected to be changed within groups, or not. Therefore, sample preparation technique and analytical method may cause dramatic differences on the results of the study [3].

Metabolites are not like DNA or proteins. The physical properties and chemical structures of metabolites are totally different within each other. The only common properties of metabolites are the fact that their molecular masses are less than $1.5 \mathrm{kDa}$ and they are organic compounds [4]. As a result of this situation, it is hard to provide a unique analytical platform to determine whole metabolites in a sample with a single injection. It is still discussed the advantages and disadvantages of sample preparation techniques prior to Liquid Chromatography-Mass Spectrometry (LC-MS) based metabolite profiling [5-8]. Although, it looks like having maximum number of metabolite peaks in a chromatogram data is the best way to obtain the most usable biological information for a sample, the real situation is not as it is expected. A mass/charge $(\mathrm{m} / \mathrm{z})$ value for a metabolite peak refers more than one metabolite in databanks within the acceptable relative error for an advanced analytical LC-MS instrument. This situation is about the fact that one $\mathrm{m} / \mathrm{z}$ ratio in an acceptable range refers more than one chemical structures. MS/MS data still have not sufficient to be evaluated through databanks. There is limited MS/MS information for already identified metabolites in databanks. Therefore, categorize the metabolites according to their polarity prior to MS detection is a good way to separate the metabolites in LC process and eliminate the possibilities on exact identification on MS. As it is already known; precipitation of proteins via organic solvents or reagents, ultrafiltration and solid phase extraction are the common sample preparation technique on LC-MS based metabolomics applications $[6,8,9]$. Precipitation of proteins and ultrafiltration are not selective sample preparation techniques against metabolites. Even though solid phase extraction is a selective technique, it is not simple and it is not as much as reproducible like others.

Grouping metabolites prior to LC-MS analyzes via sample preparation technique is a useful strategy to prevent the confusion in data mining process while it is being worked on specific disorders related with specific pathways. Especially, the diseases related with lipid metabolism disorders or the diseases cause defects on lipid metabolism could be monitored through non-polar metabolite analyzes including lipids. The plasma of healthy subjects as control group could be differentiated from the subject of interest in clinical studies through principal component analysis on metabolome level [10,11]. Thus, diagnosis of some specific diseases and monitoring the disease situation upon treatment could be successfully achieved using the semiquantitative determination of specific metabolites. Therefore, the aim of this study is to propose a liquid-liquid extraction and ultrafiltration-based sample preparation technique for LC-MS based metabolite profiling of nonpolar metabolites in human plasma samples. The proposed technique is the combination of well-known techniques previously applied on environmental analysis, lipidomics and proteomics [12-14]. A methanol/chloroform/water mixture and ultrafiltration system were used together in this application and plasma metabolites were selectively separated according to their polarity. The upper phase and lower phase on the mixture of methanol/chloroform/water system dissolved non-polar and polar metabolites, respectively. When the fractions were evaporated in vacuum-centrifuge and dissolved in an organic solvent acetonitrile, non-polar metabolites were separated into two parts and polar metabolites were discarded. A reverse phase UPLC-Q-TOF MS system in positive ionization mode was used for metabolite profiling and the number of non-polar metabolites obtained for both upper and lower phases were evaluated. The intersection of the metabolites in these two phases were identified.

The present study is the unique application for combining liquid-liquid chromatography with ultrafiltration-based extraction for especially the analysis of non-polar metabolites on untargeted metabolomic studies. The methodology and the results in this study allows researchers to specify the metabolites in the sample preparation step for their metabolite profiling based clinical studies.

\section{RESULTS}

\subsection{Chromatographic separation of metabolites}

Non-polar metabolites on upper and lower phases of the methanol/chloroform/water solvent mixture were separated in reverse phase liquid chromatography. In 25 minutes, gradient program, metabolites were separated according to their polarity. As expected, the total ion chromatogram profiles were different from each other (Figure 1). Due to the fact that final solvent was acetonitrile to dissolve the phases, relatively polar metabolites were discarded and non-polar metabolites were separated through C18 column. 

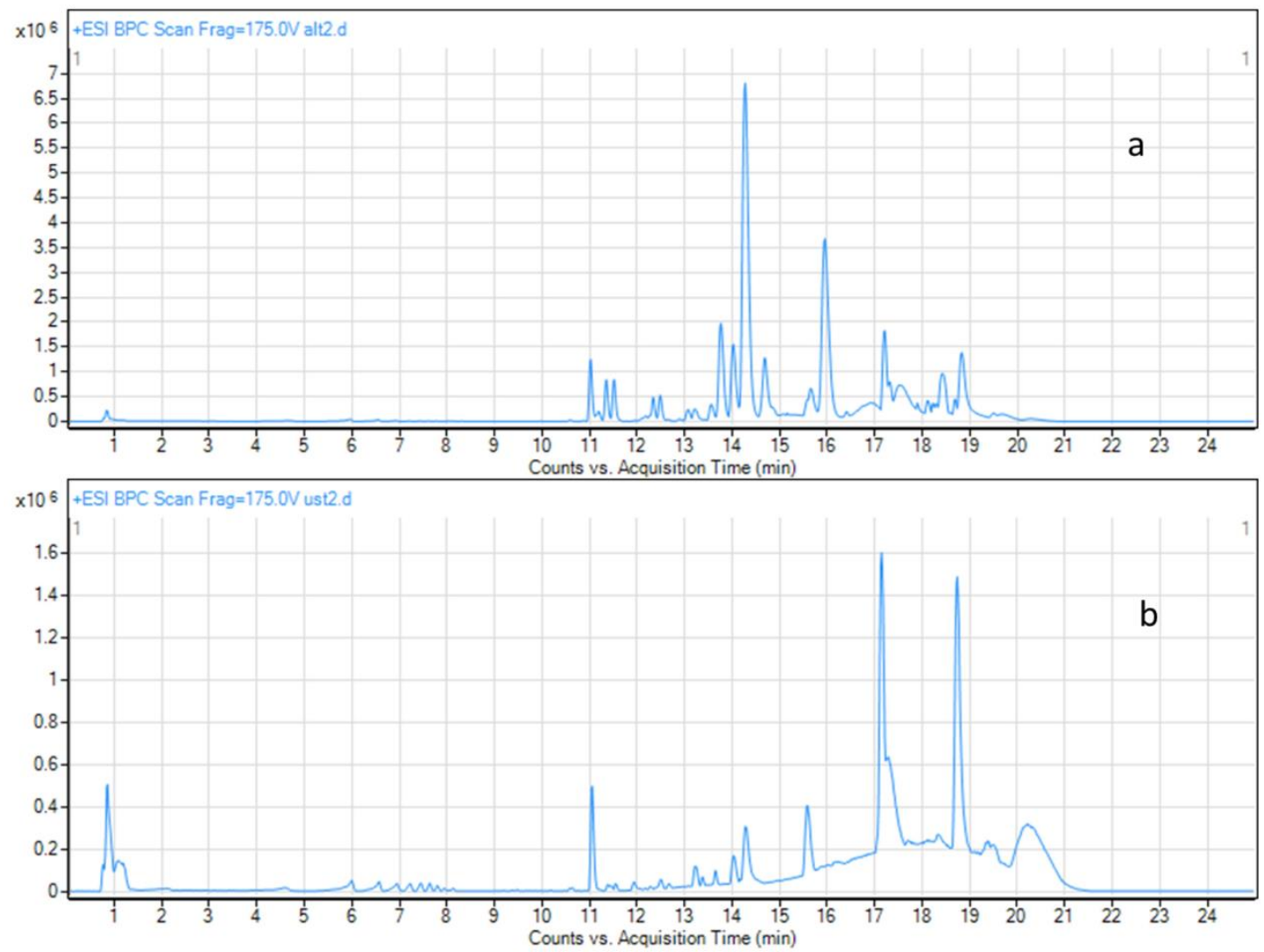

Figure 1. Total ion chromatograms of (a) lower phase (organic) and (b) upper phase (aqueous) metabolites.

\subsection{Metabolic profiling of non-polar samples}

In the present study, the main goal was to get metabolic pathway-oriented sample extraction with minimum technical variation and maximum metabolome coverage. The major advantage of the proposed method was effective protein precipitation and opportunity to study with clean sample without interference coming from matrix components. Separation of non-polar metabolites prior to LC-MS separation allows to focus on metabolites working on specific pathways related with different diseases. Methanol, water and chloroform co-solvent system has been used very commonly in proteomics research to extract proteins [15]. In this study, it was also combined solvent extraction method with ultrafiltration to clean up the sample. Metabolites were eluted and detected in reverse phase liquid chromatography according to their polarity and untargeted LC-MS-based metabolomics was performed. After removing of possible contaminants peaks through regression analysis of consecutively diluted samples, 249 and 60 reliable peaks related with non-polar metabolites were obtained in upper phase sample and lower phase, respectively. When the results were compared, 14 peaks were found as common in two phases (Figure 2).

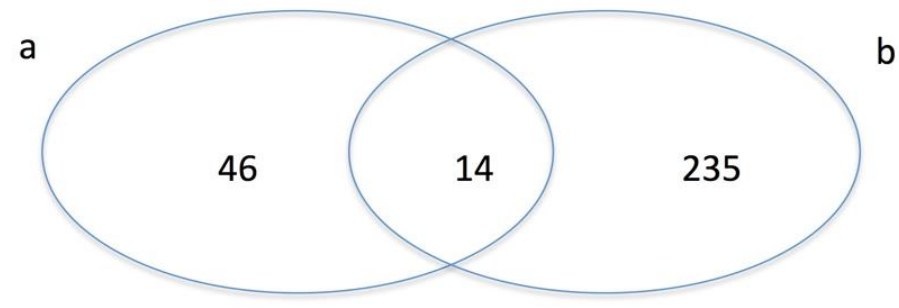

Figure 2. Obtained peak numbers for (a) lower phase (organic) and (b) upper phase (aqueous). 


\section{DISCUSSION}

In the literature, sample preparation for global metabolomics using LC/MS studies was reviewed and the data shows that average number of metabolite peaks on a single LC/MS run $(n=6)$ is between 1000 and 2000 for various sample preparation techniques combining with different chromatographic properties [16]. In the present study, total number of 295 metabolite peaks found indicate the result that the proposed sample preparation technique succesfully discarded polar metabolites and performed a specific application for nonpolar metabolites. Due to the fact that the lower phase was the aqueous phase and the polar metabolites dissolved in aqueous phase were discarded after vacumm-centrifuge of the solvent and resolvation through acetonitrile, more non-polar metabolites in organic phase (upper phase) and less non-polar metabolites in aqueous phase (lower phase) were found. As it is seen in Figure 2, the non-polar metabolites fractioned and dissolved in an organic solvent, acetonitrile, could be separated into two groups as relatively more non-polar metabolites and relatively less non-polar metabolites through the procedure proposed in this manuscript. The intersection of the non-polar metabolite groups had 14 metabolites and the separation was proven to be successful with the metabolite profiling procedure. In our opinion, the unknown 46 metabolites given in Figure 2 - cluster (a) should be searched on lipid databases where the others given in Figure 2 - cluster (b) might be searched on both lipid and metabolite clusters on specific databases. This situation presents the rigid separation between the phases may provide rigid separation on datamining processes. As a result, this study is one of the first application to extract and separate the non-polar metabolites attended in different pathways. Based on the results found in the present study, the researchers working on untargeted metabolite profiling could focus on specific non-polar metabolites in different pathways and they could monitor the disease situation according to statistically proved semi-quantitative metabolite amount comparison between groups. The methodology in the present study could be used for further investigation on metabolite profiling studies. Having an extracted and ultrafiltrated clean plasma sample including some specific metabolites is a better assay for clinical applications instead of a plasma sample including "whole" metabolites.

\section{CONCLUSION}

Metabolomics is growing area to understand cellular process at metabolome level. Integration of metabolomics data with genomics, transcriptomics and proteomics provides deeper biological understanding. Sample complexity is the main challenge for metabolomics and other omics technologies. In this study, we attended to use methanol/chloroform/water co-solvent system extraction and ultrafiltration-based sample clean-up process to profile metabolites in human plasma sample. The proposed sample preparation strategy prior to LC-MS profiling of human plasma metabolites allows researchers to focus on specific non-polar metabolites on different pathways. Dealing with specific metabolites is a useful strategy while working on some specific disorders. Comparison of two different human plasma sample subjects could be statistically found as different within each other as long as the "big" data would be converted to a useful and redefined data. Thus, the self-evident and statistically proved metabolite profiling results could be used in real life on diagnosis and monitoring the situation of some diseases.

\section{MATERIALS AND METHODS}

\subsection{Sample preparation}

Methanol (Sigma, USA), chloroform (Riedel-de Haen, Germany) and water mixture was used for separation of polar and non-polar metabolites. Firstly $400 \mu \mathrm{L}$ methanol was added into $100 \mu \mathrm{L}$ of commercial pooled plasma sample (Sigma, USA) and vortexed for $2 \mathrm{~min}$. Then, $100 \mu \mathrm{L}$ of chloroform and finally $300 \mu \mathrm{L}$ of water were added into this mixture. All chemicals were MS grade. After vortexing for 2 min, centrifugation was performed for $5 \mathrm{~min}$ at $14000 \mathrm{~g}$. After centrifugation, a three-phase system emerged. The polar metabolites were separated in the upper aqueous phase. The lower organic phase contained non-polar metabolites. In the middle of these two phases solid protein pellets were observed. The upper phase was transferred to a eppendorf tube. $400 \mu \mathrm{L}$ of methanol was added to protein and non-polar metabolites mixture. After vortexing for 2 minutes, centrifugation was carried out at $14000 \mathrm{~g}$ for 5 minutes and proteins accumulated to the bottom of the tube. Non-polar metabolites were transferred to a eppendorf tube. Upper and lower phases were filtrated using ultrafiltration membrane (Amicon ${ }^{\circledR}$ Ultra Centrifugal Filters, $0.5 \mathrm{~mL}, 3 \mathrm{kDa}$; Sigma,USA) to avoid contamination of proteins and lipids. Vacuum centrifuge system was used to remove aqueous and organic solvents. Finally, metabolites were resolved in an organic solvent, acetonitrile, and centrifuged at 5000 rpm for 10 minutes to discard if there was any unsolved component. For reliable analysis, reproducibility and 
linearity (regression analysis) parameters were evaluated. For reproducibility three replicates for each sample were prepared in vials with $40 \mu \mathrm{L}$ sample $+40 \mu \mathrm{L}$ [water:acetonitrile mixture 1:1 (v/v)] . For regression analysis samples were prepared at three different ratios $(30 \mu \mathrm{L}$ sample $+50 \mu \mathrm{L}$ [water:acetonitrile mixture 1:1 (v/v)], $20 \mu \mathrm{L}$ sample $+60 \mu \mathrm{L}$ [water:acetonitrile mixture 1:1 (v/v)] and $10 \mu \mathrm{L}$ sample $+70 \mu \mathrm{L}$ [water:acetonitrile mixture 1:1 (v/v)]). Dilution series method is one of the most important methods used to check the reliability of a peak in the data analysis section of metabolomics studies [12].

\subsection{LC/MS analysis}

The metabolic analysis was conducted on an Agilent 1290 HPLC system (Agilent, Santa Clara, CA, USA) that was coupled to a 6530 series accurate quadrupole time-of-flight mass spectrometer (Q-TOF MS) system (Agilent, Santa Clara, CA, USA). Reverse phase chromatography column used for separation (Agilent Zorbax C18 $1.8 \mu \mathrm{m}, 50 \times 2.1 \mathrm{~mm}$ ). Water and acetonitrile with consist of $0.1 \%$ formic acid both used as mobile phase for chromatography. All chemicals for mobile phase were MS grade or higher and purchased from Merck, Darmstadt, Germany. Gradient elution started with $95 \% \mathrm{H}_{2} \mathrm{O}-5 \%$ acetonitrile ratio and shifted linearly to $5 \%$ $\mathrm{H}_{2} \mathrm{O}-95 \%$ acetonitrile ratio until $13^{\text {th }}$ minute, later turned back starting ratio at $17^{\text {th }}$ minute and last step was 8 minutes post-run until $25^{\text {th }}$ minute. Flow rate was $0.200 \mathrm{ml} \mathrm{min}^{-1}$. The MS acquisition was performed in the positive-ion (ESI+) mode. The parameters for the MS acquisition were as follow: the MS capillary voltages were set at $4000 \mathrm{~V}$ in the ESI+ mode. The drying gas temperature was $350^{\circ} \mathrm{C}$, and the flow rate of the drying gas was $10 \mathrm{~L} \mathrm{~min}^{-1}$. Centroid data were collected in the full scan mode from 100 to $1700 \mathrm{~m} / \mathrm{z}$ in the positive mode with a scan rate of 1 spectra s-1.

\subsection{Data processing}

First step in data analysis operations is the process of determining and matching the peaks via XCMS software (https://xcmsonline.scripps.edu/) software. The raw data files received from LC/MS instrument have been converted to .mzml format via ProteoWizard software http:/ / proteowizard.sourceforge.net). Then, optimized $\mathrm{xcms}$ parameters were determined by using IPO software and XCMS analysis was performed with these optimized parameters. The results obtained were subjected to regression analysis according to the dilution series method and the peaks without $\mathrm{R}>0.90$ were filtered. This operation allows us to extract pollutants or noise peaks and to consider "reliable" peaks as metabolites.

Author contributions: Concept - M.Ç., E.K., O.K.; Design - M.Ç., E.K.; Supervision - M.Ç.; Materials - M.Ç., E.K., O.K.; Data Collection and/or Processing - E.K., O.K.; Analysis and/or Interpretation -E.K., O.K.; Literature Search - E.K., M.Ç.; Writing - E.K., M.Ç.; Critical Reviews - M.Ç., E.K., O.K.

Conflict of interest statement: The authors declared no conflict of interest

\section{REFERENCES}

[1] Beger RD. A review of applications of metabolomics in cancer. Metabolites. 2013; 3(3): 552-574. [CrossRef]

[2] Dunn WB. Current trends and future requirements for the mass spectrometric investigation of microbial, mammalian and plant metabolomes. Phys Biol. 2008; 5(1): 011001. [CrossRef]

[3] Dunn WB, Erban A, Weber RJ, Creek DJ, Brown M, Breitling R, et al. Mass appeal: metabolite identification in mass spectrometry-focused untargeted metabolomics. Metabolomics. 2013; 9(1): 44-66. [CrossRef]

[4] Alonso A, Marsal S, Julià A. Analytical methods in untargeted metabolomics: state of the art in 2015. Front Bioeng Biotechnol. 2015; 3: 23. [CrossRef]

[5] Bruce SJ, Tavazzi I, Parisod Vr, Rezzi S, Kochhar S, Guy PA. Investigation of human blood plasma sample preparation for performing metabolomics using ultrahigh performance liquid chromatography/mass spectrometry. Anal Chem. 2009; 81(9): 3285-3296. [CrossRef]

[6] Vuckovic D. Current trends and challenges in sample preparation for global metabolomics using liquid chromatography-mass spectrometry. Anal Bioanal Chem. 2012;403(6): 1523-1548. [CrossRef]

[7] Gika H, Theodoridis G. Sample preparation prior to the LC-MS-based metabolomics/metabonomics of bloodderived samples. Bioanalysis. 2011; 3(14): 1647-1661. [CrossRef]

[8] Tulipani S, Llorach R, Urpi-Sarda M, Andres-Lacueva C. Comparative analysis of sample preparation methods to handle the complexity of the blood fluid metabolome: When less is more. Anal Chem. 2012; 85(1): 341-348. [CrossRef] 
[9] Fernández-Peralbo M, de Castro ML. Preparation of urine samples prior to targeted or untargeted metabolomics mass-spectrometry analysis. Trac-Trend Anal Chem. 2012; 41: 75-85. [CrossRef]

[10] Worley B, Powers RJCM. Multivariate analysis in metabolomics. Curr Metabolomics. 2013;1(1): 92-107. [CrossRef]

[11] Hendriks MM, van Eeuwijk FA, Jellema RH, Westerhuis JA, Reijmers TH, Hoefsloot HC, et al. Data-processing strategies for metabolomics studies. Trac-Trend Anal Chem. 2011;30(10): 1685-98. [CrossRef]

[12] González Fernández-Niño SM, Smith-Moritz AM, Chan LJ, Adams PD, Heazlewood JL, Petzold CJ. Standard flow liquid chromatography for shotgun proteomics in bioenergy research. Front Bioeng Biotechnol. 2015; 3: 44. [CrossRef]

[13] Feist P, Hummon AB. Proteomic challenges: sample preparation techniques for microgram-quantity protein analysis from biological samples. Int J Mol Sci. 2015; 16(2): 3537-3563. [CrossRef]

[14] Dutta A, Shetty P, Bhat S, Ramachandra Y, Hegde S. A mass spectrometric study for comparative analysis and evaluation of metabolite recovery from plasma by various solvent systems. J Biomol Tech. 2012; 23(4): 128-135. [CrossRef]

[15] Eliasson M, Rännar S, Madsen R, Donten MA, Marsden-Edwards E, Moritz T, et al. Strategy for optimizing LC-MS data processing in metabolomics: a design of experiments approach. Anal Chem. 2012; 84(15): 6869-6876. [CrossRef]

[16] Vuckovic D. Current trends and challenges in sample preparation for global metabolomics using liquid chromatography-mass spectrometry. Anal Bioanal Chem. 2012; 403: 1523-1548. [CrossRef]

This is an open access article which is publicly available on our journal's website under Institutional Repository at http://dspace.marmara.edu.tr. 